\title{
Invited article:
}

\section{HALL EFFECTS ON ROTATING MHD CHANNEL FLOW IN THE PRESENCE OF AN} INCLINED MAGNETIC FIELD

\author{
S. Das ${ }^{1}$, B.C. Sarkar ${ }^{2}$, R. N. Jana ${ }^{3}$ and A. J. Chamkha ${ }^{4, *}$ \\ ${ }^{1}$ Department of Mathematics, University of Gour Banga, Malda 732 103, India \\ ${ }^{2,3}$ Department of Applied Mathematics, Vidyasagar University, Midnapore 721 102, India \\ ${ }^{4}$ Mechanical Engineering Department, Prince Mohammad Bin Fahd University (PMU) Al-Khobar 31952, Kingdom of Saudi Arabia \\ *E-mail: achamkha@pmu.edu.sa
}

\begin{abstract}
MHD flow of a viscous incompressible electrically conducting fluid between two parallel plates in a rotating system in the presence of an inclined magnetic field has been studied on taking Hall current into account. An exact solution of the governing equations has been obtained in closed form. Numerical results of the fluid velocity components and the shear stresses at the plates are being discussed graphically. It is observed that both Hall currents as well as the angle of inclination of the applied magnetic field have a retarding influence on the primary fluid velocity whereas they accelerate the secondary fluid velocity. The electric field components are being calculated and presented in tabular form. Asymptotic behavior of the solution has been analyzed for small as well as large values of magnetic parameter and rotation parameter. It is interesting to note that either for strong magnetic field or for large rotation there exists a single-deck boundary layer near the upper plate.
\end{abstract}

Keywords: MHD flow, Hall current, channel flow, rotating fluid, angle of inclination.

AMS Subject Classification: 76W05, 76R10

\section{INTRODUCTION}

MHD flow in a rotating system has received wide attention to many researchers due to its varied and wide applications in many areas of science and technology. The rotating flow of an electrically conducting fluid in the presence of a magnetic field is encountered in Geographical fluid dynamics. It is also important in the solar physics dealing with the sunspot development, the solar cycle and the structure of rotating magnetic stars. It is well known that a number of astronomical bodies possess fluid interiors and magnetic fields. Changes that take place in the rate of rotation, suggest the possible importance of hydromagnetic spin-up. The hydromagnetic flow in a rotating channel with Hall effects has been discussed by Seth and Ghosh [1]. Ghosh $[2,3]$ has studied the Hall effects on MHD flow in a rotating channel permeated by an inclined magnetic field in the presence of an oscillator. Hall effects on MHD plasma flow in a rotating environment in the presence of an inclined magnetic field have been analysed by Ghosh and Pop [4, 5]. Mondal et al. [6] have studied the Hall effects on MHD plasma Couette flow in a rotating system. The Hall effects in the viscous flow of an ionized gas 
between parallel plates under transverse magnetic field have been examined by Sato [7]. An analysis has been made on an oscillatory Couette flow in the presence of an inclined magnetic field by Guria et al.[8]. Seth et al.[9] have studied the MHD Couette flow in a rotating system in the presence of inclined magnetic field. Seth and Ghosh $[10,11]$ have initiated the study of the hydromagnetic flow in a rotating channel in the presence of an inclined magnetic field neglecting induced magnetic field. The steady Hartmann flow of a viscous incompressible electrically conducting fluid in a rotating channel in the presence of an inclined magnetic field taking induced magnetic field into account considering different aspects of the problem has been investigated by Ghosh and Bhattacharjee [12]. Seth et al.[13] have studied the Hydromagnetic flow in a rotating channel in the presence of an inclined magnetic field. Hall effects on oscillatory hydromagnetic Couette flow in a rotating system have been observed by Seth et al.[14]. Hall effects on MHD Couette flow between two infinite horizontal parallel porous plates in a rotating system under the boundary layer approximations have been studied by Das et al.[15]. Effects of Hall current on oscillatory hydromagnetic couette flow of class-II in a rotating system in the presence of an inclined magnetic field have been investigated by Seth et al.[16]. Recently, Seth et al.[17] have studied the unsteady hydromagnetic Couette flow of a viscous incompressible electrically conducting fluid in a rotating system in the presence of an inclined magnetic field taking Hall currents into account. Ghosh and Pop [18] have studied the MHD plasma behavior of a rotating environment between two infinite parallel plates channel in the presence of inclined magnetic field. In the formulation of the problem they have assumed that for continuous medium $\nabla \cdot \vec{j} \neq 0$, that is, the conservation of the electric current does not hold. This assumption is seemed to be incorrect. The conservation of electric current must hold good for any conducting fluid. Further, the equation (2.4) of Ghosh and Pop [18] shows that the electric field is non-zero as $\vec{E} \equiv\left(E_{x}, E_{y}, E_{z}\right)$. But equations (2.5) - (2.7) of Ghosh and Pop [18] show that electric field as well as induced magnetic field are zero. Hence the equation (2.4) of Ghosh and Pop [18] are not compatible with the fundamental equations of MHD as they have stated. In the present study we have reconsidered the problem studied by Ghosh and Pop[18]. We have obtained the solution of the problem where electric field need not be equal to zero $(\vec{E} \neq 0)$ and the conservation of electric current $(\nabla \cdot \vec{j}=0)$ holds. The effects of the Hall current, the rotation and the angle of inclination of the magnetic field on both the velocity distribution have been studied. It is seen that the Hall current and the angle of inclination retard the primary velocity while they have an accelerating influence on the secondary velocity. The rotation has retarding influence on both the primary velocity and the secondary velocity. Asymptotic behavior of the solution is analyzed for small as well as large values of magnetic parameter $M^{2}$ and rotation parameter $K^{2}$ to gain some physical insight into the flow pattern. It is found that a thin boundary layer is formed near the upper plate, for large values of rotation parameter $K^{2}$. The thickness of this boundary layer decreases with increase in magnetic parameter $M^{2}$.

\section{MATHEMATICAL FORMULATION AND PROBLEM SOLUTION}

The basic equations of magnetohydrodynamics for steady flow are:

$$
\begin{gathered}
(\vec{q} \cdot \nabla) \vec{q}+2 \Omega \hat{k} \times \vec{q}=-\frac{1}{\rho} \nabla p+\nu \nabla^{2} \vec{q}+\frac{1}{\rho} \vec{j} \times \vec{B}, \\
\nabla \cdot \vec{q}=0,
\end{gathered}
$$




$$
\begin{aligned}
& \nabla \times \vec{B}=\mu_{e} \vec{j}, \\
& \nabla \times \vec{E}=0, \\
& \nabla \cdot \vec{B}=0, \\
& \nabla \cdot \vec{D}=\rho_{e},
\end{aligned}
$$

together with generalised Ohm's law on taking Hall current into account is (see Cowling [19])

$$
\vec{j}+\frac{\omega_{e} \tau_{e}}{B_{0}}(\vec{j} \times \vec{B})=\sigma(\vec{E}+\vec{q} \times \vec{B})
$$

Where $\vec{q}, \vec{B}, \vec{E}, \vec{j}$ and $\vec{D}$ are respectively the velocity vector, the magnetic field vector, the electric field vector, the current density vector and the displacement vector. Also, $\sigma, v, \mu_{e}$, $\rho, \rho_{e}, p, B_{0}, \omega_{e}$ and $\tau_{e}$ are respectively the electric conductivity, kinematic viscosity, magnetic permeability, fluid density, charge density, modified fluid pressure including centrifugal force, applied magnetic field, cyclotron frequency and electron collision time. Consider the steady hydromagnetic flow of a viscous incompressible electrically conducting fluid between parallel plates at $z= \pm L$, rotating with a uniform angular velocity $\Omega$ about an axis perpendicular to the plates. The plates and the fluid rotate in unison with uniform angular velocity $\Omega$. A uniform magnetic field ${ }^{B_{0}}$ is applied at an angle $\theta$ with the vertical as shown in Figure 1. Since the plates are infinitely long along $x$ and $y$ directions, all physical quantities, except pressure, will be function of $z$ only. It is assumed that the induced magnetic field may be neglected in comparison to the applied magnetic field since the magnetic Reynolds number is very small for liquid metals for partially ionized gases. The solenoid relation $\nabla \cdot \vec{B}=0$ gives $B_{z}=B_{0} \cos \theta$ everywhere in the fluid where $\vec{B} \equiv\left(B_{0} \sin \theta, 0, B_{0} \cos \theta\right)$.The conservation of electric current $\nabla \cdot \vec{j}=0$ yields $j_{z}^{\prime}=$ constant where $\vec{j} \equiv\left(j_{x}^{\prime}, j_{y}^{\prime}, j_{z}^{\prime}\right)$.

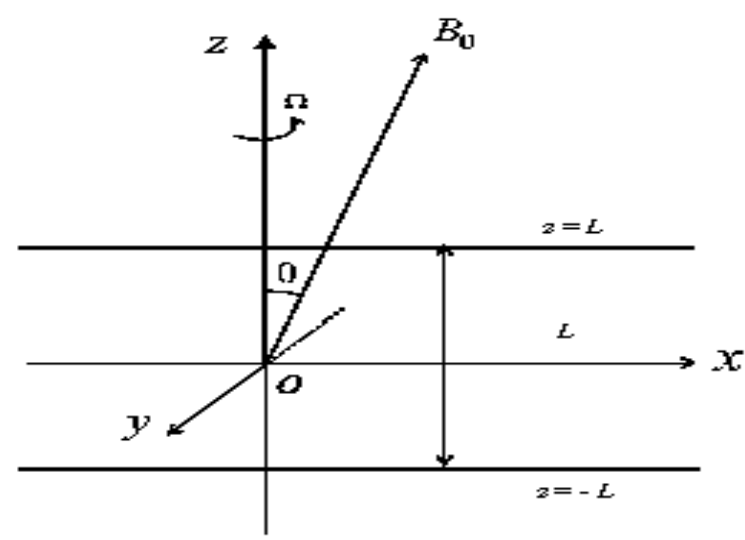

Fig.1. Geometry of the problem

This constant is zero since $j_{z}^{\prime}=0$ at the plates which are electrically non-conducting. Hence, $j_{z}^{\prime}=0$ everywhere in the flow. Since the motion is steady, we have from Maxwell equation $\nabla \times \vec{E}=0$ which in turn yields $E_{x}=$ constant and $E_{y}=$ constant everywhere in the flow. In view of the above assumptions, the equation (7) yields ;

$j_{x}^{\prime}+m j_{y}^{\prime} \cos \theta=\sigma\left[E_{x}+B_{0} v^{\prime} \cos \theta\right]$,

$j_{y}^{\prime}-m j_{x}^{\prime} \cos \theta=\sigma\left[E_{y}-B_{0} u^{\prime} \cos \theta\right]$,

$-m j_{y}^{\prime} \sin \theta=\sigma\left[E_{z}-B_{0} v^{\prime} \sin \theta\right]$.

Solving for $j_{x}^{\prime}$ and $j_{y}^{\prime}$ from (8) and (9), we have;

$j_{x}^{\prime}=\frac{\sigma}{1+m^{2} \cos ^{2} \theta}\left[\left(E_{x}-m E_{y} \cos \theta\right)+B_{0} \cos \theta\left(v^{\prime}+m u^{\prime} \cos \theta\right)\right],(11)$

$j_{y}^{\prime}=\frac{\sigma}{1+m^{2} \cos ^{2} \theta}\left[\left(E_{y}+m E_{x} \cos \theta\right)+B_{0} \cos \theta\left(m v^{\prime} \cos \theta-u^{\prime}\right)\right]$.

On the use of (11) and (12), the equations of motion in a rotating frame of reference are;

$-2 \Omega v^{\prime}=-\frac{1}{\rho} \frac{\partial p}{\partial x}+v \frac{d^{2} u^{\prime}}{d z^{2}}$ 
$+\frac{\sigma B_{0} \cos \theta}{\rho\left(1+m^{2} \cos ^{2} \theta\right)}\left[\left(E_{y}+m E_{x} \cos \theta\right)+B_{0} \cos \theta\left(m v^{\prime} \cos \theta-u^{\prime}\right)\right]$,

$2 \Omega u^{\prime}=v \frac{d^{2} v^{\prime}}{d z^{2}}-\frac{\sigma B_{0} \cos \theta}{\rho\left(1+m^{2} \cos ^{2} \theta\right)}\left[\left(E_{x}-m E_{y} \cos \theta\right)+B_{0} \cos \theta\left(v^{\prime}+m u^{\prime} \cos \theta\right)\right]$,

$0=-\frac{1}{\rho} \frac{\partial p}{\partial z}-\frac{\sigma B_{0} \sin \theta}{\rho\left(1+m^{2} \cos ^{2} \theta\right)}\left[\left(E_{y}+m E_{x} \cos \theta\right)+B_{0} \cos \theta\left(m v^{\prime} \cos \theta-u^{\prime}\right)\right]$.

The boundary conditions are

$u^{\prime}=v^{\prime}=0$ at $z= \pm L$.

Introducing the non-dimensional variables

$\eta=\frac{z}{L},\left(u^{\prime}, v^{\prime}\right)=(u, v) \frac{v}{L},\left(E_{x}, E_{y}, E_{z}\right)=\frac{v B_{0}}{L}\left(e_{x}, e_{y}, e_{z}\right),\left(j_{x}^{\prime}, j_{y}^{\prime}\right)=\frac{\sigma v B_{0}}{L}\left(j_{x}, j_{y}\right)$,

equations (13)-(15) become

$-2 K^{2} v=R+\frac{d^{2} u}{d \eta^{2}}+\frac{M^{2} \cos \theta}{1+m^{2} \cos ^{2} \theta}\left[\left(e_{y}+m e_{x} \cos \theta\right)+\cos \theta(m v \cos \theta-u)\right]$,

$2 K^{2} u=\frac{d^{2} v}{d \eta^{2}}-\frac{M^{2} \cos \theta}{1+m^{2} \cos ^{2} \theta}\left[\left(e_{x}-m e_{y} \cos \theta\right)+\cos \theta(v+m u \cos \theta)\right]$,

$0=-\frac{\partial p^{*}}{\partial \eta}-\frac{M^{2} \sin \theta}{1+m^{2} \cos ^{2} \theta}\left[\left(e_{y}+m e_{x} \cos \theta\right)+\cos \theta(m v \cos \theta-u)\right]$,

where $M^{2}=\frac{\sigma B_{0}^{2} L^{2}}{\rho \nu}$ is the magnetic parameter, $K^{2}=\frac{\Omega L^{2}}{v}$ the rotation parameter, $R=\left(-\frac{\partial p^{*}}{\partial x}\right)$ and $p^{*}=\frac{L^{2} p}{\rho v^{2}}$.

The boundary conditions (16) become

$$
u=v=0 \text { at } \eta= \pm 1 \text {. }
$$

Equations (18) and (19) can be solved subject to the boundary conditions (21) and the solution can be written as

$$
\begin{aligned}
& u=\frac{1}{2}\left\{\frac{1}{(\alpha+i \beta)^{2}}\left[R+\frac{E_{0} M^{2}(1+i m \cos \theta) \cos \theta}{\left(1+m^{2} \cos ^{2} \theta\right)}\right]\left[1-\frac{\cosh (\alpha+i \beta) \eta}{\cosh (\alpha+i \beta)}\right]\right. \\
& \left.+\frac{1}{(\alpha-i \beta)^{2}}\left[R+\frac{\bar{E}_{0} M^{2}(1-i m \cos \theta) \cos \theta}{\left(1+m^{2} \cos ^{2} \theta\right)}\right]\left[1-\frac{\cosh (\alpha-i \beta) \eta}{\cosh (\alpha-i \beta)}\right]\right\}, \\
& v=\frac{i}{2}\left\{\frac{1}{(\alpha-i \beta)^{2}}\left[R+\frac{\bar{E}_{0} M^{2}(1-i m \cos \theta) \cos \theta}{\left(1+m^{2} \cos ^{2} \theta\right)}\right]\left[1-\frac{\cosh (\alpha-i \beta) \eta}{\cosh (\alpha-i \beta)}\right]\right. \\
& \left.-\frac{1}{(\alpha+i \beta)^{2}}\left[R+\frac{E_{0} M^{2}(1+i m \cos \theta) \cos \theta}{\left(1+m^{2} \cos ^{2} \theta\right)}\right]\left[1-\frac{\cosh (\alpha+i \beta) \eta}{\cosh (\alpha+i \beta)}\right]\right\},
\end{aligned}
$$

where $E_{0}=e_{y}-i e_{x}$,

$\alpha, \beta=\frac{1}{\sqrt{2}}\left[\left(a_{1}^{2}+b_{1}^{2}\right)^{\frac{1}{2}} \pm a_{1}\right]^{\frac{1}{2}}, a_{1}=\frac{M^{2} \cos ^{2} \theta}{1+m^{2} \cos ^{2} \theta}, b_{1}=2 K^{2}+\frac{m M^{2} \cos ^{3} \theta}{1+m^{2} \cos ^{2} \theta}$

and $\bar{E}_{0}$ is the complex conjugate of $E_{0}$.

On the use of (17) and combining equations

(11) and (12), we have

$$
J=\frac{1+i m \cos \theta}{1+m^{2} \cos ^{2} \theta}\left(E_{\mathrm{o}}-F \cos \theta\right),
$$

where $J=j_{y}-i j_{x}, \quad F=u+i v$.

\section{RESULTS AND DISCUSSION}

To study the effects of Hall current, rotation and the angle of inclination of the magnetic field on the velocity field, the numerical values of the fluid velocity are shown graphically against $\eta$ for several values of Hall parameter $m$, rotation parameter $K^{2}$ and angle of inclination $\theta$ of the magnetic field in Figures 2 to 4 when $M^{2}=10$ and $R=1$. It is seen from Figure 2 that the primary velocity $u$ decreases with an increase in rotation parameter $K^{2}$.

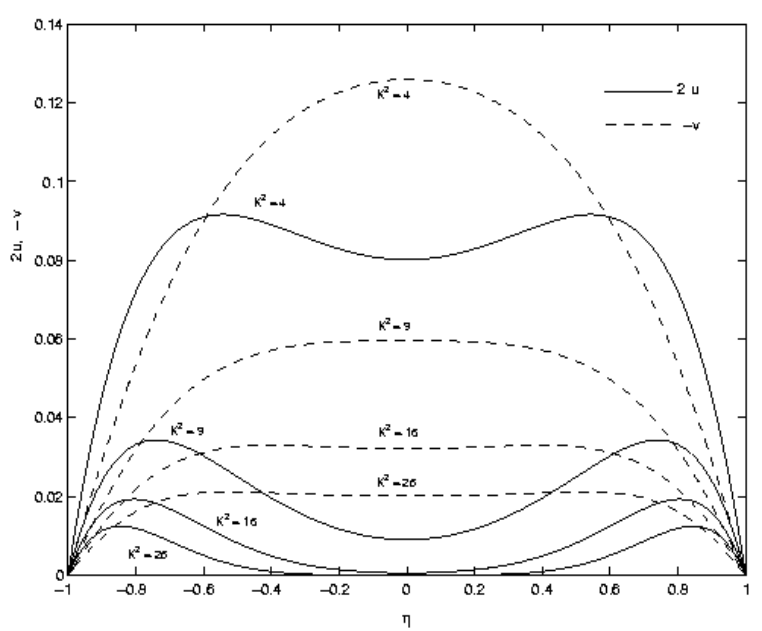

Fig. 2. Primary and secondary velocities for different $K^{2}$ when $\theta=45^{\circ}$ and $m=0.5$

The primary velocity $u$ is more near the plates than that at the middle of the channel. 


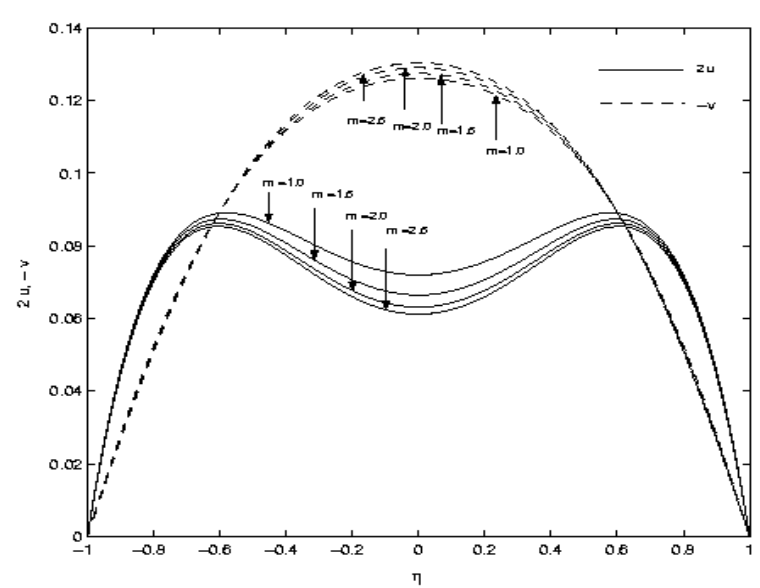

Fig. 3. Primary and secondary velocities for different $m$ when $\theta=45^{\circ}$ and $K^{2}=4$

Further, the hump near the plates exhibits the formation of the boundary layer in the vicinity of the plates. It is also seen that the magnitude of the secondary velocity $v$ decreases with an increase in rotation parameter $K^{2}$. This means that the rotation has retarding influence on both primary and secondary flow. It is observed from Figure 3 that the primary velocity $u$ decreases while the magnitude of secondary velocity $v$ increases with an increase in Hall parameter $m$. It means that Hall current has a retarding influence on the primary velocity while it accelerates the secondary velocity.

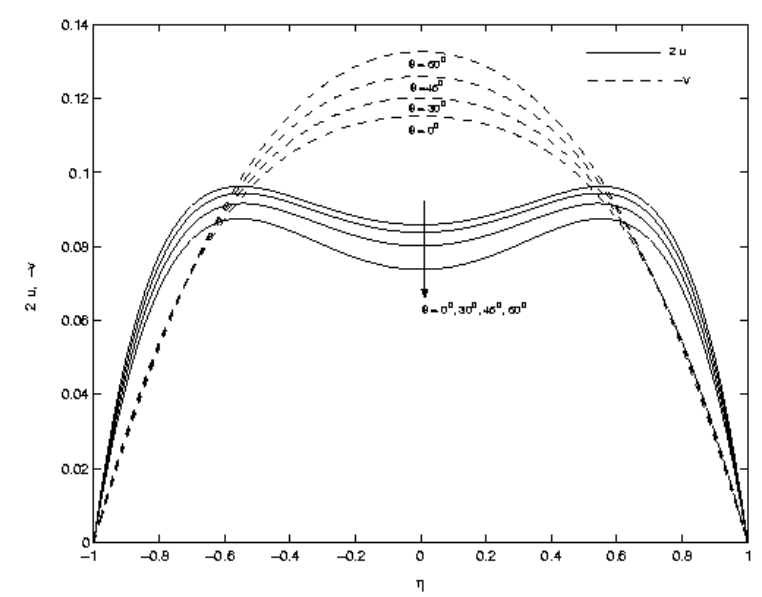

Fig. 4. Primary and secondary velocities for different $\theta$ when $m=0.5$ and $K^{2}=4$

It is also observed from Figure 4 that the primary velocity $u$ decreases while the magnitude of the secondary velocity $v$ increases with an increase in the angle of inclination $\theta$ of the magnetic field. This implies that the angle of inclination has a retarding influence on the primary velocity while it accelerates the secondary velocity. To determine the electric field $E_{0}$, we impose the condition

$$
\int_{-1}^{1} J d \eta=0
$$

which is equivalent to the assumption that the total current flowing between the plates is zero. On the use of (25), together with (22) and (23) in (26), integrating and rearranging, we get

$e_{x}=\frac{i}{2}\left\{\frac{R \cos \theta[(\alpha+i \beta)-\tanh (\alpha+i \beta)]}{\left[(\alpha+i \beta)^{2}-2 i K^{2}\right] \tanh (\alpha+i \beta)+2 i K^{2}(\alpha+i \beta)}\right.$

$$
\left.-\frac{R \cos \theta[(\alpha-i \beta)-\tanh (\alpha-i \beta)]}{\left[(\alpha-i \beta)^{2}+2 i K^{2}\right] \tanh (\alpha-i \beta)-2 i K^{2}(\alpha-i \beta)}\right\}
$$

$e_{y}=\frac{1}{2}\left\{\frac{R \cos \theta[(\alpha+i \beta)-\tanh (\alpha+i \beta)]}{\left[(\alpha+i \beta)^{2}-2 i K^{2}\right] \tanh (\alpha+i \beta)+2 i K^{2}(\alpha+i \beta)}\right.$

$$
\left.+\frac{R \cos \theta[(\alpha-i \beta)-\tanh (\alpha-i \beta)]}{\left[(\alpha-i \beta)^{2}+2 i K^{2}\right] \tanh (\alpha-i \beta)-2 i K^{2}(\alpha-i \beta)}\right\} .
$$

It is observed from Tables 1 and 2 that the electric field component $e_{x}$ first decreases, reach a minimum and then increases while the electric field component $e_{y}$ decreases with an increase in Hall parameter $m$. It is also seen that both $e_{x}$ and $e_{y}$ decrease with an increase in either rotation parameter $K^{2}$ or the angle of inclination $\theta$ of the magnetic field.

\subsubsection{Case I}

When $M^{2}=1$ and $K^{2}=1$, then equations (22) and (23) become

$$
\begin{aligned}
& u(\eta)=\frac{1}{2}\left(1-\eta^{2}\right)\left[R+\frac{M^{2} \cos \theta\left(e_{y}+m e_{x} \cos \theta\right)}{1+m^{2} \cos ^{2} \theta}\right] \\
& -\frac{R}{24} \frac{M^{2} \cos ^{2} \theta}{1+m^{2} \cos ^{2} \theta}\left(\eta^{4}-6 \eta^{2}+5\right)+\cdots,
\end{aligned}
$$




$$
\begin{aligned}
& v(\eta)=\frac{1}{2}\left(1-\eta^{2}\right) \frac{M^{2} \cos \theta}{1+m^{2} \cos ^{2} \theta}\left(e_{y} m \cos \theta-e_{x}\right) \\
& -\frac{R}{24}\left(2 K^{2}+\frac{m M^{2} \cos ^{3} \theta}{1+m^{2} \cos ^{2} \theta}\right)\left(\eta^{4}-6 \eta^{2}+5\right)+\cdots
\end{aligned}
$$

It is interesting to note that for small values of $M^{2}$ and $K^{2}$, the primary velocity $u(\eta)$ is unaffected by rotation whereas the secondary velocity $v(\eta)$ is affected by both the magnetic field and rotation. If $e_{x}=e_{y}=0$ then equations (29) and (30) become

$$
\begin{aligned}
& u(\eta)=\frac{R}{2}\left(1-\eta^{2}\right)-\frac{R}{24} \frac{M^{2} \cos ^{2} \theta}{1+m^{2} \cos ^{2} \theta}\left(\eta^{4}-6 \eta^{2}+5\right)+\cdots, \\
& v(\eta)=-\frac{R}{24}\left(2 K^{2}+\frac{m M^{2} \cos ^{3} \theta}{1+m^{2} \cos ^{2} \theta}\right)\left(\eta^{4}-6 \eta^{2}+5\right)+\cdots
\end{aligned}
$$

Further, if $M^{2}=0$ and $K^{2}=0$, then equations (31) and (32) reduce to

$$
u(\eta)=\frac{R}{2}\left(1-\eta^{2}\right) \text { and } v(\eta)=0 .
$$

Equation (33) gives the hydrodynamical flow between infinite parallel plates channel in the presence of an applied pressure gradient.

\subsubsection{Case II}

When $M^{2}=1$ and $K^{2} ? 1$. In this case equations (22) and (23) yield

$$
\begin{aligned}
& u(\eta)=\frac{1}{2 K^{2}}\left[(R+A) e^{-\alpha_{1}(1-\eta)} \sin \beta_{1}(1-\eta)+B\left(1-e^{-a_{1}(1-\eta)} \cos \beta_{1}(1-\eta)\right)\right], \\
& v(\eta)=\frac{1}{2 K^{2}}\left[B e^{-a_{1}(1-\eta)} \sin \beta_{1}(1-\eta)-(R+A)\left(1-e^{-q_{1}(1-\eta)} \cos \beta_{1}(1-\eta)\right)\right],
\end{aligned}
$$

Where;

$\alpha_{1}, \beta_{1}=K\left[1 \pm \frac{M^{2} \cos ^{2} \theta}{4 K^{2}\left(1+m^{2} \cos ^{2} \theta\right)}(1 \pm m \cos \theta)\right]$

$A=\frac{M^{2} \cos \theta}{1+m^{2} \cos ^{2} \theta}\left(e_{y}+m e_{x} \cos \theta\right), B=\frac{M^{2} \cos \theta}{1+m^{2} \cos ^{2} \theta}\left(e_{y} m \cos \theta-e_{x}\right)$.

It is seen from the above equations that there exists a thin boundary layer near the plate $\eta=1$. The thickness of this boundary layer is of the order of $\mathrm{O}\left(\alpha_{1}^{-1}\right)$ where $\alpha_{1}$ is given by (36). The thickness of this boundary layer decreases with an increase in the magnetic parameter while it increases with an increase in rotation

\begin{tabular}{|c|c|c|c|c|c|c|c|c|}
\hline & \multicolumn{4}{|c|}{$e_{x}$} & \multicolumn{4}{|c|}{$e_{y}$} \\
\hline$m \backslash K^{2}$ & 4 & 9 & 16 & 25 & 4 & 9 & 16 & 25 \\
\hline 0.0 & 0.06424 & 0.03297 & 0.01943 & 0.01277 & 0.02841 & 0.00748 & 0.00298 & 0.00149 \\
\hline 0.5 & 0.06312 & 0.03267 & 0.01935 & 0.01274 & 0.02719 & 0.00741 & 0.00298 & 0.00149 \\
\hline 1.0 & 0.06285 & 0.03249 & 0.01930 & 0.01272 & 0.02591 & 0.00724 & 0.00295 & 0.00148 \\
\hline 1.5 & 0.06304 & 0.03243 & 0.01927 & 0.01271 & 0.02497 & 0.00707 & 0.00291 & 0.00147 \\
\hline 2.0 & 0.06370 & 0.03243 & 0.01927 & 0.01271 & 0.02435 & 0.00695 & 0.00288 & 0.00146 \\
\hline
\end{tabular}
parameter, since $\alpha_{1}$ increases with an increase in the magnetic parameter but decreases with an increases in rotation parameter. If $e_{x}=e_{y}=0$ then the equations (34) and (35) become

TABLE 1: ELECTRIC FIELD COMPONENTS $e_{x}$ AND $e_{y}$ WHEN $\boldsymbol{M}^{2}=10$ AND $\boldsymbol{\theta}=\mathbf{4 5 ^ { \circ }}$ 
TABLE 2: ELECTRIC FIELD COMPONENTS $\boldsymbol{e}_{x}$ AND $\boldsymbol{e}_{y}$ WHEN $\boldsymbol{M}^{2}=\mathbf{1 0}$ AND $\boldsymbol{K}^{2}=\mathbf{4}$

\begin{tabular}{|c|c|c|c|c|c|c|c|c|}
\hline & \multicolumn{9}{|c|}{$e_{x}$} & \multicolumn{3}{c|}{$e_{y}$} \\
\hline$m \backslash \theta$ & $0^{0}$ & $30^{0}$ & $45^{\circ}$ & $60^{0}$ & $0^{0}$ & $30^{0}$ & $45^{0}$ & $60^{0}$ \\
\hline 0.0 & 0.08696 & 0.07703 & 0.06424 & 0.04627 & 0.04550 & 0.03728 & 0.02841 & 0.01840 \\
0.5 & 0.08459 & 0.07520 & 0.06312 & 0.04589 & 0.04089 & 0.03457 & 0.02719 & 0.01812 \\
1.0 & 0.08507 & 0.07517 & 0.06285 & 0.04569 & 0.03738 & 0.03218 & 0.02591 & 0.01776 \\
1.5 & 0.08624 & 0.07581 & 0.06304 & 0.04564 & 0.03539 & 0.03067 & 0.02497 & 0.01742 \\
2.0 & 0.08734 & 0.07651 & 0.06337 & 0.04568 & 0.03430 & 0.02978 & 0.02435 & 0.01715 \\
\hline
\end{tabular}

$u(\eta)=\frac{R}{2 K^{2}} e^{-\alpha_{1}(1-\eta)} \sin \beta_{1}(1-\eta)$,

$v(\eta)=-\frac{R}{2 K^{2}}\left[1-e^{-\alpha_{1}(1-\eta)} \cos \beta_{1}(1-\eta)\right]$.

It is seen that the exponential terms in (34) and (35) damp out quickly as $\eta$ increases. When $\eta \geq 1 / \alpha_{1}$, i.e. outside the boundary layer region, the primary and secondary velocities become

$$
\begin{aligned}
& u=\frac{M^{2} \cos \theta\left(e_{y} m \cos \theta-e_{x}\right)}{2 K^{2}\left(1+m^{2} \cos ^{2} \theta\right)}, \\
& v=-\frac{1}{2 K^{2}}\left[\frac{M^{2} \cos \theta\left(e_{y}+m e_{x} \cos \theta\right)}{2 K^{2}\left(1+m^{2} \cos ^{2} \theta\right)}+R\right] .
\end{aligned}
$$

Further, if $e_{x}=e_{y}=0$ then from equations (40) and (41), we have

$$
u(\eta)=\mathrm{O} \text { and } v(\eta)=-\frac{R}{2 K^{2}} .
$$

\subsubsection{Case III}

When $M^{2}=1$ and $K^{2}=1$, then equations (22) and (23) become

$$
\begin{aligned}
& u(\eta)=(R C+A C+B D)\left[1-e^{-\alpha_{2}(1-\eta)} \cos \beta_{2}(1-\eta)\right] \\
& +(R D-B C+A D) e^{-\alpha_{2}(1-\eta)} \sin \beta_{2}(1-\eta), \\
& v(\eta)=(R C+A C+B D) e^{-\alpha_{2}(1-\eta)} \sin \beta_{2}(1-\eta) \\
& -(R D-B C+A D)\left[1-e^{-\alpha_{2}(1-\eta)} \cos \beta_{2}(1-\eta)\right\rfloor,
\end{aligned}
$$

where

$$
\begin{array}{ll}
\alpha_{2}=\frac{M \cos \theta}{\sqrt{1+m^{2} \cos ^{2} \theta}}, & \beta_{2}=\frac{1}{2} \frac{\sqrt{1+m^{2} \cos ^{2} \theta}}{M \cos \theta}\left(2 K^{2}+\frac{m M^{2} \cos ^{3} \theta}{1+m^{2} \cos ^{2} \theta}\right), \\
C=\frac{1+m^{2} \cos ^{2} \theta}{M^{2} \cos ^{2} \theta}, & D=\frac{m}{M^{2} \cos \theta}\left(1+m^{2} \cos ^{2} \theta\right) .
\end{array}
$$

Like Case II, in this case also there exists a thin boundary layer near the plate $\eta=1$. The thickness of this boundary layer is of order of $\mathrm{O}\left(\alpha_{2}^{-1}\right)$ where $\alpha_{2}$ is given by (45). The thickness of this boundary layer decreases with an increase in magnetic parameter $M^{2}$ whereas it increases with an increase in Hall parameter $m$. It is interesting to note that the boundary layer thickness is unaffected by the rotation when magnetic field is very strong. In the absence of $e_{x}$ and $e_{y} \quad\left(e_{x}=e_{y}=0\right)$, equations (43) and (44) become

$$
\begin{aligned}
& u(\eta)=\frac{R\left(1+m^{2} \cos ^{2} \theta\right)}{M^{2} \cos ^{2} \theta}\left[1-e^{-\alpha_{2}(1-\eta)} \cos \beta_{2}(1-\eta)\right] \\
& +\frac{R m}{M^{2} \cos \theta}\left(1+m^{2} \cos ^{2} \theta\right) e^{-\alpha_{2}(1-\eta)} \sin \beta_{2}(1-\eta),
\end{aligned}
$$

$$
\begin{aligned}
& v(\eta)=\frac{R\left(1+m^{2} \cos ^{2} \theta\right)}{M^{2} \cos ^{2} \theta} e^{-\alpha_{2}(1-\eta)} \sin \beta_{2}(1-\eta) \\
& -\frac{R m}{M^{2} \cos \theta}\left(1+m^{2} \cos ^{2} \theta\right)\left[1-e^{-\alpha_{2}(1-\eta)} \cos \beta_{2}(1-\eta)\right] .
\end{aligned}
$$

Further, the exponential terms in equations (46) and (47) damp out quickly as $\eta$ increases near $\eta \geq 1 / \alpha_{2}$ where $\alpha_{2}$ is given by (45). The 
fluid flow outside the boundary layer is given by

$u=\frac{R\left(1+m^{2} \cos ^{2} \theta\right)}{M^{2} \cos ^{2} \theta}, v=-\frac{R m}{M^{2} \cos \theta}\left(1+m^{2} \cos ^{2} \theta\right)$.

In this case also the fluid flow behavior is similar to Case II. The non-dimensional shear stress components $\tau_{x}$ and $\tau_{y}$ at the plates $(\eta=-1)$ and $(\eta=1)$ due to the primary and secondary flows are respectively given by

$\tau_{x}=\left(\frac{d u_{1}}{d \eta}\right)_{\eta=\mp 1}= \pm \frac{[\{\alpha(R+A)+\beta B\} \sinh 2 \alpha+\{\beta(R+A)-\alpha B\} \sin 2 \beta]}{\left(\alpha^{2}+\beta^{2}\right)(\cosh 2 \alpha+\cos 2 \beta)}$,

$\tau_{y}=\left(\frac{d v_{1}}{d \eta}\right)_{\eta=\mp 1}= \pm \frac{[\{\alpha B-\beta(R+A)\} \sinh 2 \alpha+\{\alpha(R+A)+\beta B\} \sin 2 \beta]}{\left(\alpha^{2}+\beta^{2}\right)(\cosh 2 \alpha+\cos 2 \beta)}$,

where $\alpha, \beta$ are given by (24) and $A, B$ are given by (37). Numerical results of the shear stress components $\tau_{x}$ and $\tau_{y}$ are being presented in the Figures 5 to 6 for several values of $m, K^{2}$ and $\theta$ when $M^{2}=10$ and $R=1$. Figure 5 and 6 show that both the magnitude of the shear stresses $\tau_{x}$ and $\tau_{y}$ decrease with an increase in either $K^{2}$ or $\theta$. It is illustrated from Figure 6 that the magnitude of the shear stress $\tau_{x}$ first increases, reach as a maximum and then it decreases while the shear stress $\tau_{y}$ decreases with an increase in Hall parameter $m$.

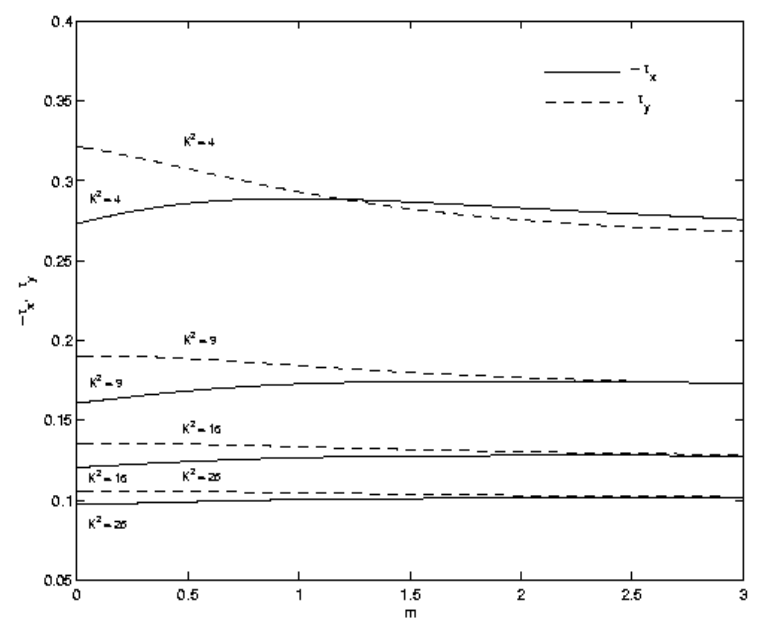

Fig.5. Shear stresses $\tau_{x}$ and $\tau_{y}$ for different $K^{2}$ when $\theta=45^{\circ}$

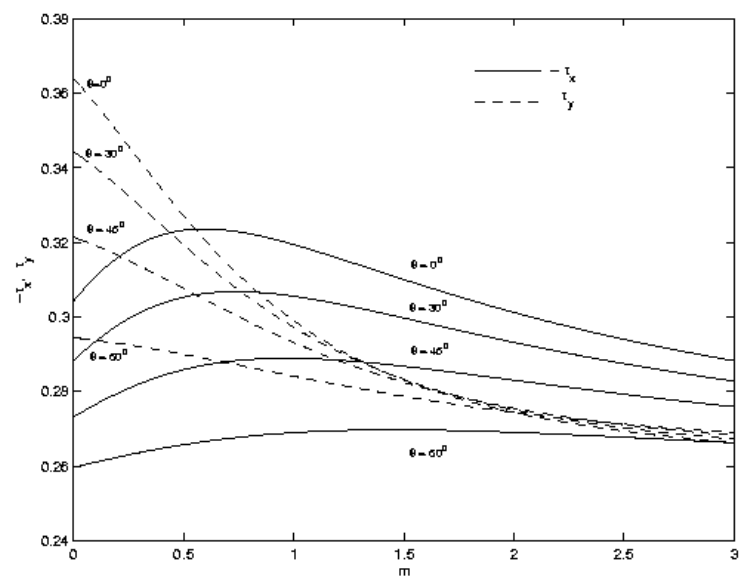

Fig. 6. Shear stresses $\tau_{x}$ and $\tau_{y}$ for different $\theta$ when $K^{2}=4$

\section{CONCLUSION}

Hall effects on MHD flow permeated by an inclined magnetic field in a rotating system have been investigated. An exact solution of the governing equations describing the flow has been obtained in closed form. Hall currents and angle of inclination of the applied magnetic field have a retarding influence on the primary velocity while they have an accelerating influence on the secondary velocity. Asymptotic behavior of the solution has been analyzed for small as well as large values of magnetic parameter $M^{2}$ and rotation parameter $K^{2}$ to gain some physical insight into the flow pattern. The thickness of this boundary layer decreases with an increase in magnetic parameter $M^{2}$. Further, both the electric field components decrease with an increase in inclination of the applied magnetic field with the axis of rotation. The magnitude of thr shear stresses $\tau_{x}$ and $\tau_{y}$ at the plate decrease with an increase in either rotation parameter $K^{2}$ or the angle of inclination $\theta$.

\section{REFERENCES}

[1] G.S. Seth and S.K. Ghosh, Hydromagnetic flow in a rotating channel with Hall effects, Acta Ciencia Indica, XXV M(4) (1999), 403-406.

[2] S.K. Ghosh, Hall effect on unsteady hydromagnetic flow in a rotating channel 
permeated by an inclined magnetic field in the presence of an oscillator, Czechoslovak J. Physics, 49(4) (1999a), 465-472.

[3] S.K.Ghosh, Hall effect on unsteady hydromagnetic flow in a rotating channel permeated by an inclined magnetic field in the presence of an oscillator (Erratum), Czechoslovak J. Physics, 49(12) (1999b), 1751.

[4] S.K.Ghosh and I. Pop, Hall effects on MHD plasma Couette flow in a rotating environment, Int. J. Applied Mechanics and Engineering, 9(2) (2004), 293-305.

[5] S.K.Ghosh and I. Pop, Comments on MHD plasma flow in a rotating environment in the presence of an inclined magnetic field, Int. J. Applied Mechanics and Engineering, 9(4) (2004), 821-826.

[6] G. Mondal, K.K.Mandal and G. Choudhury, Hall effects on MHD plasma Couette flow in a rotating system, J. Phys. Soc. Jpn., 51(1982), 2010.

[7] H. Sato, The Hall effects in the viscous flow of ionized gas between parallel plates under transverse magnetic field, J. Phys. Soc. Jpn., 16(7) (1961), 1427-1433.

[8] M.Guria, S. Das, R.N. Jana and S.K.Ghosh, Oscillatory Couette flow in the presence of an inclined magnetic field, Meccanica, 44(2009), 555-564.

[9] G.S. Seth, R. Nandkeolyar, N. Mahto and S.K.Singh, MHD Couette flow in a rotating system in the presence of inclined magnetic field, Appl. Math. Sci., 3(59) (2009), 2919-2932.

[10] G.S. Seth and S.K. Ghosh, Unsteady hydromagnetic flow in a rotating channel in the presence of oblique magnetic field, Int. J. Engng. Sci., 24(1986), 1183.
[11] G.S.Seth and S.K. Ghosh, Hydromagnetic flow in a rotating channel in the presence of inclined magnetic field, Proc. Math. Soc., BHU, 11(1995), 111.

[12] S.K. Ghosh, and P.K. Bhattacharjee, Hall effects on steady hydromagnetic flow in a rotating channel in the presence of an inclined magnetic field, Czech. J. Phys., 50 (2000), 759.

[13] G. S. Seth, Md. S. Ansary, N. Mahto and S.K. Singh, Hydromagnetic flow in a rotating channel in the presence of an inclined magnetic field, Acta Ciencia Indica, XXXIV M(3) (2008), 1279-1288

[14] G. S. Seth, R. Nandkeolyar and Md. S. Ansari, Hall effects on oscillatory hydromagnetic Couette flow in a rotating system, Int. J. Acad. Res., 1(2009), 6-17.

[15] S. Das, B. C. Sarkar and R. N. Jana, Hall effects on MHD Couette flow in rotating system, Int. J Com. Appl., 35(13) (2011), 22-30

[16] G. S. Seth, G. K. Mahato and R. Nandkeolyar, Effects of Hall current on oscillatory hydromagnetic couette flow of class-II in a rotating system in the presence of an inclined magnetic field, Int. J. Adv. Sci. and Tech. Res., 1(1)(2011), 31-47.

[17] G. S. Seth, R. Nandkeolyar and Md. S. Ansari, Effects of Hall current and rotation on unsteady MHD Couette flow in the presence of an inclined magnetic field, J. Appl. Flu. Mech., 5(2) (2012), 67-74.

[18] S.K. Ghosh and I. Pop, An analytical approach to MHD plasma behavior of a rotating environment in the presence of an inclined magnetic field as compared to excitation frequency, Int. J. Applied Mechanics and Engineering, 11(4) (2006), 845856. T. G. Cowling, Magnetohydrodynamics, New york, Intersscience, 1957. 


\title{
تأثيرات HALL على معدل سريان قناة MHD الدورانية في وجود حقل مغناطيسي مائل
}

\author{
اس. داس1، بي. سي. ساركار2، ار. إن. جانا3، إيه. جيه. شامخا4 \\ 1 قسم الرياضيات، جامعة جور بانجا، مالدا 732103، الهند \\ 2, 3 قسم الرياضيات التطبيقية، جامعة فيدياساجار، مادنابور 721102، الهند
}

4 قسم الهندسة الميكانيكية، جامعة الامير محمد بن فهد، الخبر 31952، المملكة العربية السعودية

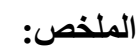

تمت دراسة معدل تدفق MHD لسائل لزج غير قابل للضغط وموصل كهربياً بين لوحتين منو ازيتين في نظام دو ار في وجود مجال مغناطيسي مائل مع أخذ تيار Hall في الاعتبار. كما تم التوصل إلى حل دقيق للمعادلات الحاكمة في شكل مغلق. ان النتائج العددية لمكونات سرعة السائل وإجهاد القص في اللوحات تمت مناقثتها بيانيا. ولقد لوحظ أن كلا من تبار Hall فضلا عن زاوية ميل المجال المغناطيسي المطبق لها تأثيثر مثبط على سرعة السائل الأولية في حين أنها تزيد سرعة السائل الثانوية. ولقد نم حساب مكونات المجال الكهربائي وعرضها في شكل جداول. كما تم تحليل السلوك المتناظر من الحل لقيم صغيرة وكذلك قيم كبيرة من عوامل المغناطيسية وعو امل الدوران. ومن المثير للاهتمام أن نلاحظ أن سواء تم استخدام حقل مغناطيسي قوي أو دوران كبير فان هناك طبقة احادية السطح تتكون بالقرب من اللوحة العلوية. 